\title{
Leveraging the Entirety of Protein Data Bank to Enable Improved Structure Prediction based on
}

Cross-Link Data

Andrew Keller ${ }^{1}$, Juan D. Chavez ${ }^{1}$, Xiaoting Tang ${ }^{1}$, and James E. Bruce ${ }^{1^{*}}$

${ }^{1}$ Department of Genome Sciences, University of Washington, Seattle, WA 98105, USA

jimbruce@uw.edu

\section{Supporting Information}

Table of Contents (list of supplementary components)

- Figure S1: Examples of three cross-links that could not be mapped to a structure with homologous sequences.

- Predicted reduction in fraction of false positive interprotein cross-links upon mapping to multi-protein structures.

- Table S1: Details of the mappings of the 72 cross-linked protein residue pairs identified in mouse heart mitochondria ${ }^{20}$ to specific chains within the structure 5ARA of bovine mitochondrial ATP synthase state 1 a.

- Training tutorial on how to view structures mapped to cross-links on XLinkDB. 


\begin{tabular}{|c|c|c|c|c|c|c|c|c|c|}
\hline $\begin{array}{c}\text { Cross- } \\
\text { link }\end{array}$ & $\begin{array}{c}\text { Sample } \\
\text { organism }\end{array}$ & Protein1 & Residue1 & Protein2 & Residue2 & Structure & Chain & $\begin{array}{c}\text { Structure } \\
\text { organism }\end{array}$ & Reason for not mapping \\
\hline 1 & M.musculus & P97450 & 94 & 0.03265 & 531 & $6 \mathrm{~J} 5$ & $\mathrm{C}$ & S.scrofa & $\begin{array}{l}\text { Amino acid in structure corresponding to } \\
\text { P97450 residue } 94 \text { is threonine, not lysine }\end{array}$ \\
\hline 2 & M.musculus & P03930 & 48 & Q9D3D9 & 136 & $6 \mathrm{~J} 5 \mathrm{I}$ & 8 & S.scrofa & $\begin{array}{l}\text { Amino acid in structure corresponding to } \\
\text { P03930 residue 48 is serine, not lysine }\end{array}$ \\
\hline 3 & M.musculus & 035143 & 98 & $\mathrm{Q} 03265$ & 531 & $10 \mathrm{HH}$ & $\mathrm{H}$ & B.taurus & $\begin{array}{l}\text { O35143 residues beyond } 72 \text { not present in } \\
\text { structure }\end{array}$ \\
\hline
\end{tabular}

Figure S1: Examples of three cross-links that could not be mapped to a structure with homologous sequences.

\section{Predicted reduction in fraction of false positive interprotein cross-links upon mapping to multi-} protein structures.

Let there be a cross-link dataset with interprotein cross-links consisting of $\mathrm{N}_{\mathrm{TP}}$ true positives and $\mathrm{N}_{\mathrm{FP}}$ false positives. Let $\mathbf{t}$ and $\mathbf{f}$ be the fractions of true positive and false positive interprotein cross-links, respectively, that are mapped to structures. The factor $\mathbf{t}$ is determined by the set of proteins cross-linked in a sample as well as the available structures for mapping. For the average dataset on XLinkDB with $1 \%$ FDR, $\mathbf{t}$ is equal to 0.33 reflecting it having close to one third of its true-positive interprotein cross-links mapped to structures. In contrast, the factor $\mathbf{f}$ is largely determined by how likely a cross-link's two peptides correspond by chance to distinct components of a multi-protein structure. If one assumes an average proteome size of 10,000 proteins and an average number of proteins per structure as 10 , then the chance that a false positive interprotein cross-link will be mapped at both ends to a common structure will be on the order of $10 / 10,000$ or $10^{-3}$. Since $\mathbf{f}$ as well as $\mathbf{t}$ is limited by the available structures, $\mathbf{f}$ is estimated to be less than $10^{-3}$.

The FDR among all interprotein cross-links is equal to $\mathrm{N}_{\mathrm{FP}} /\left(\mathrm{N}_{\mathrm{TP}}+\mathrm{N}_{\mathrm{FP}}\right)$. After mapping, there are expected to be $\mathrm{tN}_{\mathrm{TP}}$ true positives and $\mathrm{fN}_{\mathrm{FP}}$ false positives with an FDR among mapped interprotein cross-links, FDR mapped, equal to $f N_{F P} /\left(t N_{T P}+f N_{F P}\right)$. Substituting $\left(N_{F P}-F D R * N_{F P}\right) / F D R$ for the value of $\mathrm{N}_{T P}$ yields:

$$
\frac{F D R^{\text {mapped }}}{F D R}=\frac{\boldsymbol{f}}{\boldsymbol{f} F D R+\boldsymbol{t}-\boldsymbol{t} F D R}
$$


The FDR among mapped interprotein cross-links, FDR mapped, is less than the FDR among all interprotein cross-links when:

$$
\boldsymbol{f}<\boldsymbol{f} F D R+\boldsymbol{t}-\boldsymbol{t} F D R
$$

Rearranging yields:

$$
\boldsymbol{f}(1-F D R)<\boldsymbol{t}(1-F D R)
$$

The fraction of interprotein cross-links that are false positive will thus be reduced upon mapping to structures provided that FDR $<1$ and $\mathbf{f}<\mathbf{t}$. As discussed above, $\mathbf{f}$ is expected to be much less than $\mathbf{t}$ under most circumstances. Thus, as long as there are some true positive interprotein cross-links present in a dataset, they are expected to be enriched among those mapped to multiprotein structures, with FDR ${ }^{\text {mapped }}<$ FDR. Furthermore, the number of interprotein cross-links mapped to structures that are false positive is estimated to be effectively zero as long as either FDR is 0 or $f<1 / F D R\left(N_{T P}+N_{F P}\right)$. It should be emphasized that mapping is not recommended as a substitute for employing a validated method to control FDR since mapping interprotein crosslinks with a high FDR will less likely lead to a satisfactorily low FDR ${ }^{\text {mapped. }}$ 